\title{
Glycated Hemoglobin Level is Significantly Associated with The Severity of Ischemic Stroke in Libyan Non- Diabetic and Diabetic Patients
}

\author{
Asgad A Abdalgbar ${ }^{1,2}$ and Hanan GK Altalhi ${ }^{1 *}$ \\ ${ }^{1}$ Department of Internal Medicine, Omer Elmaukhtar University, Africa \\ ${ }^{2}$ Faculty of Medical Technology, Omer Elmaukhtar University, Africa
}

*Corresponding author: Hanan GK Altalhi, Department of Internal Medicine, Faculty of Medicine, Omer Elmaukhtar University, Elbaida Libya, Africa.

To Cite This Article: Hanan GK Altalhi. Glycated Hemoglobin Level is Significantly Associated with The Severity of Ischemic Stroke in Libyan Non-Diabetic and Diabetic Patients. Am J Biomed Sci \& Res. 2019 - 2(6). AJBSR.MS.ID.000615. DOI: 10.34297/AJBSR.2019.02.000615

Received: March 29, 2019 | Published: May 06, 2019

\begin{abstract}
Background: Diabetes is metabolic disorder that lead to atherosclerosis which could lead to vascular accident like acute ischemic stroke. To formulate effective preventive measures, it's important to understand the impact of glycemic status on the severity of acute ischemic stroke in adult type II diabetic patient.
\end{abstract}

Aim of study: Study to analyze the role of HbA1c level on the severity of acute ischemic stroke in adult over 39-year diabetic type II Libyan patients.

Patients and methods: This is case control study of 40 subjects with acute ischemic stroke and diabetes type II (case subjects) were categorized in to 2 sub-groups based on $\mathrm{HbA} 1 \mathrm{c}$ level at time of admission with acute ischemic stroke, patients with diabetes and acute ischemic stroke with good glycemic control (<7HbA1c) and patient with acute ischemic stroke and diabetes with poor glycemic control ( $>7 \mathrm{HbA} 1 \mathrm{c}) .40$ acute ischemic stroke patients without diabetes as controls, the diagnosis of cerebral infarction was confirmed by brain - CT scan. Neurological impairment was evaluated using the National Institutes of Health Stroke Scale (NIHSS). All subjects have blood hemoglobin A1c (HbA1c) measured at admission.

Result: Poor glycemic control group had statistically significant high HbA1c value (8.510 1.260$)$ when compared to good glycemic control group (5.921 \pm 0.501$)$ and non-diabetic $(5.8 \pm 0.540)$. Stroke severity at admission were higher in patients with poor glycemic control than in those with good glycemic control (90\% vs $46.33 \%$ ) with good glycemic control.

Conclusion: Glycemic control has significant association on severity of ischemic stroke patients with diabetes. Estimation of HbA1c level at the time of admission might be predictor of the severity of neurological impairment in patient with acute ischemic stroke and diabetes mellitus type II.

Keywords: Hemoglobin A1c; Stroke; Diabetes mellitus; Brain - CT

\section{Introduction}

Stroke is a condition that results in high mortality rate and severe disabilities. Most stroke survivors can do experience improvements in their functional abilities, but the amount, rate, timing, pattern, type, and ultimate outcome of the improvements differ across patients and situation [1]. Numerous studies were conducted on the prognostic factors of acute ischemic stroke. Stroke severity and patient age are the main predictors of stroke outcome in acute phase [2,3].

Diabetes mellitus is one of the established risk factors for stroke. It has been demonstrated that diabetic patients had worse residual neurological deficits and functional outcome when compared with non-diabetic patients [4-6]. Hyperglycemia occurred in up to one - third of patients with acute ischemic stroke was associated with higher mortalities independent of age and stroke severity $[7,8]$.

Hemoglobin A1c has direct relationship with mean glycemia because erythrocytes are continuously glycated during their 120 -day lifespan, which means that the rate of formation of glycated hemoglobin, also called HbA1c, is proportional to the ambient glucose concentration [9]. In the diabetes control and complication trial, an $\mathrm{HbA1c}$ of $6 \%$ corresponded to a mean serum glucose level of $135 \mathrm{mg} / \mathrm{dl}$, and the measurement of $\mathrm{HbA1c}$ has been the primary index of glycemia [9]. An HbA1c test can be used to diagnose pre 
-diabetes or diabetes and check the long-term control of blood glucose levels in people with diabetes. Serum blood glucose level changes during the day for many reasons, including medicine, diet, exercise. And the level of insulin in the blood. While the HbA1c test result is not affected by any recent changes [10].

The aim of this study was to elucidate the association between glycemic control status, defined by HbA1c on admission and the severity of acute ischemic stroke in diabetic type II Libyan patients.

\section{Aims and Objectives}

To know the glycemic status by estimating the HbA1c, fasting and postprandial plasma glucose at admission among patients with acute ischemic stroke. To study the effect of HbA1c on the severity of acute ischemic stroke at admission in patients with diabetes type II and without diabetes. To compare the poor and good glycemic status patients among the diabetics for the competence of glycemic status on the severity of stroke.

\section{Patients and Methods}

This Is case control study of 40 patients admitted to hospital with diagnosis of acute ischemic stroke with diabetes as cases.

Cases (All acute ischemic stroke patients with diabetes) were categorized into 2 subgroups based on HbA1c level at time of admission as acute ischemic stroke patient with diabetes with good glycemic control (<7HbA1c) and acute ischemic stroke patients with poor glycemic control (>7HbA1c). 40 acute ischemic stroke patients without diabetes as control, were over age 39. Data were collected through interview case and controls, physical and neurological examination by study physicians.

The diagnosis of cerebral infarction was confirmed by Brain-CT scan. Echocardiography was done to exclude heart failure. ECG was done to exclude Atrial fibrillation. Stroke severities were evaluated by using the National Institute of Health Stroke Scale (NIHSS) score on admission. The NIHSS is a tool used to objectively quantify the impairment caused by stroke and is composed of 11 items: level of consciousness, horizontal eye movement, visual field test, facial palsy. Motor arm, motor leg, limb ataxia, sensory, language, speech, extinction and inattention. Each of these specific abilities is given score between 0 and 4 . For each item, a score of (0) indicates normal function, (1 - 4) mild stroke, (5-15) moderate stroke<) 15 ) sever stroke [11].

Glycemic status in acute ischemic stroke patients was evaluated by measuring the $\mathrm{HbA} 1 \mathrm{c}$, fasting and post prandial plasma glucose were recorded for all individual.HbA1c level were recorded at time of stroke when patient initially admitted, FBG, PPG, BP and lipid profile values were taken from the data base of the registry of these patients, height and weight were measured and body mass index was calculated by dividing the weight $(\mathrm{kg})$ by the height $\left(\mathrm{m}^{2}\right)$ The exclusion criteria were intracerebral hemorrhage, space occupying lesion, subarachnoid hemorrhage, cerebral venous thrombosis, transient ischemic attack, atrial fibrillation, acute myocardial infarction, heart failure, end stage renal failure.

\section{Other various risk factors}

Bp measurement, cigarette smoking to be quantified based on daily consumption and duration of smoking. Blood biochemistry analysis was made; cholesterol, TG, LDL, HDL, CBC, RFT.

\section{Statistical analysis}

Was performed by using SPPS software (version 11), difference among groups was analyzed by (t- test) and $\mathrm{P}$ value 0.05 was considered significant.

Result

\begin{tabular}{|c|c|c|c|c|}
\hline Variable & GGC (\%) N:30 & PGC (\%) N:10 & Non-Diabetic (\%) N:40 & P Value \\
\hline Age.mean \pm SD & $61.43 \pm 59.90$ & $59.11 \pm 11.59$ & $62.87 \pm 12.397$ & NS \\
\hline Males n (\%) & $10(66.7)$ & $8(80.0)$ & $29(72.5)$ & NS \\
\hline Females n(\%) & $20(33.3)$ & $2(20.0)$ & $11(27.5)$ & NS \\
\hline HTN (\%) & $19(63.3)$ & $6(60)$ & $21(53.3)$ & NS \\
\hline Smoking & $9(30)$ & $6(60)$ & $13(33.3)$ & 0.008 \\
\hline BMI $\left(\mathrm{kg} / \mathrm{m}^{2}\right)$ & $22.23 \pm 2.34$ & $24.23 \pm 2.34$ & $23.95 \pm 2.12$ & NS \\
\hline TGL (mg/dl) & $141 \pm 56.086$ & $182.50 \pm 77.027$ & $130.3 \pm 44.11$ & 0.0001 \\
\hline LDL (mg/dl) & $102.9 \pm 34.086$ & $113.08 \pm 42.34$ & $104.2 \pm 3142$ & NS \\
\hline HDL (mg/dl) & $44.02 \pm 11.42$ & $46.35 \pm 15.77$ & $44.67 \pm 13.11$ & NS \\
\hline $\mathrm{TC}(\mathrm{mg} / \mathrm{dl})$ & $188.9 \pm 39.299$ & $209 \pm 46.67$ & $173.2 \pm 46.7$ & 0,005 \\
\hline FPG (mg/dl) & $103 \pm 24.5$ & $160.28 \pm 55.45$ & $96 \pm 22.15$ & 0,0001 \\
\hline HbA1c (\%) & $5.921 \pm 0.501$ & $8.510 \pm 1.260$ & $5.8 \pm 0.540$ & 0.0001 \\
\hline
\end{tabular}

GGC: Good Glycemic Control; PGC: Poor Glycemic Control; Non - diabetic, Control group; BMI: Body Mass Index; TGL: Triglyceride; LDL: Low Density Lipoprotein; HDL: High Density Lipoprotein; TC: Total Cholesterol; FPG: Fasting Plasma Glucose; PPG: Post Prandial Plasma Glucose; HbA1c: Glycated Hemoglobin

(Table 1) 80 patients were included in the study, 40 patients with acute ischemic stroke and diabetes (case subjects). They

were grouped into good glycemic control $<7 \mathrm{HbA1c}(\mathrm{No}=30)$, poor glycemic control $>7 \mathrm{HbA1c}(\mathrm{No}=10) .40$ patient $\mathrm{s}$ with acute 
ischemic stroke without diabetes (control subjects). Mean age was $59.90 \pm 11.3$ in the case subjects with poor glycemic control, $61.43 \pm 10.7$ in the case subjects with good glycemic control and $61.03 \pm 14.6$ in the control subjects. There was no difference in mean age, sex distribution and frequency of hypertension among groups. All smokers were males without significant difference among groups.

Patients of poor glycemic control had high total cholesterol, TGL, values when compared to good glycemic control and nondiabetic groups $(\mathrm{P}<0.05)$. Despite high mean $\mathrm{LDL}$ values noted in poor glycemic control patients, it had no statistically significant correlation. High mean HDL values were noted in good glycemic control patients and it had statistical correlation among three groups.

Glycemic status indicators like FPG, PPG, HbA1c had very high statistical significance (0.0001) values among 3 groups of patients. High mean FPG of $160.28 \pm 55.45$ was noted in poor glycemic control groups when compared to $103 \pm 24.5$ in good glycemic control and $96 \pm 22.15$ in non-diabetic group. PPG also had similar trend of statistical distribution among 3 groups with high mean PPG of $208.53 \pm 65.22$ was noted in poor glycemic group. Poor glycemic control group had statistically significant high HbA1c value 8.510 \pm 1.260 when compared to good glycemic control group $(5.8 \pm 0.5$ ) and non-diabetics $(5.6 \pm 0.4)$.

\begin{tabular}{|c|c|c|c|c|}
\hline Table 2: Stroke severity at admission (NIHSS) in cases vs. control group. \\
\hline NIHSS & Score & GGC N (\%) & PGC N (\%) & Non -diabetic \\
\hline Mild & 4 -Jan & $16(53.33)$ & $1(10)$ & $26(65 \%)$ \\
\hline Moderate & $15-$-May & $13(43.33)$ & $5(50 \%)$ & $13(32.5 \%)$ \\
\hline sever & $<16$ & $1(3.33)$ & $4(40)$ & $1(2.5)$ \\
\hline
\end{tabular}

(Table 2) Stroke severity had very high statistical significance (0.0001) values among three groups of patients. (90\%) of poor glycemic control group had moderate to severe stroke (> 7NIHSS) at admission when compared to (46.63\%) in good glycemic control and $(38.3 \%)$ in non-diabetic group.
(Table 3) TGL and glycemic indicators like FPG, PPG and HbA1c were having positive statistical correlation between the two groups. Poor glycemic control group had high stroke severity at admission when compared to good glycemic control group patients.

\begin{tabular}{|c|c|c|c|}
\hline \multirow{2}{*}{ Table 3: Baseline, Descriptive statistic for good glycemic control (GGC) and poor Glycemic control (PGC). } & PGC & \multirow{2}{*}{ P value } \\
\cline { 2 - 4 } Variable & GGC & NO:10 & $160.28 \pm 55.54$ \\
\hline FPG & $103 \pm 24.5$ & $208.53 \pm 65.22$ & 0.001 \\
\hline PPG & $130.5 \pm 38.08$ & $8.510 \pm 1.260$ & 0.0001 \\
\hline HbA1C & $5.921 \pm 0.501$ & $11.8 \pm 4.480$ & 0.0001 \\
\hline NIHSS & $6.5 \pm 2.313$ & & 0.0001 \\
\hline
\end{tabular}

\begin{tabular}{|c|c|c|c|}
\hline \multirow{2}{*}{ Table 4: Comparison between patients with good glycemic control and non-diabetic patients. } & PGC & \multirow{2}{*}{ P value } \\
\cline { 2 - 4 } Variable & GGC & NO:10 & 0.69 \\
\hline FBG (mg/dl) & $103.5 \pm 24.5$ & $96 \pm 22.15$ & 0.42 \\
\hline PPG (mg/dl) & $130.5 \pm 38.08$ & $120.31 \pm 28.97$ & 0.56 \\
\hline HbA1C & $5.921 \pm 0.5015$ & $5.8 \pm 0.540$ & 0.07 \\
\hline NIHSS & $6.5 \pm 2.313$ & $8.71 \pm 311$ & \\
\hline
\end{tabular}

(Table 4) There were no statistically significant associations between the two groups regarding glycemic indicators (FPG, PPG, $\mathrm{HbA1c}$ ). Interesting finding to be noted was stroke severity are not different among the patients of good glycemic control group and non-diabetic group.

\section{Discussion}

An increased HbA1c level reflects poor long-term glycemic control and has its specific implications on the structure and function of vascular bed including small as large cerebral vessel. Increased HbA1c level might also be marker of poor compliance indicating an unhealthy life style.
Evidence is compelling that increased stroke risk is associated with high levels of total cholesterol and low-density lipoprotein and decreased high density lipoprotein [12]. In the present study also results shown that high mean levels of total cholesterol $(\mathrm{P}<0.005)$ and Triglycerides $(\mathrm{P}<0.0001)$ levels in patients of ischemic stroke with poor glycemic control when compared with good glycemic control and non-diabetics ischemic stroke patient $[6,13]$.

Kizer JR et al. [14] studied the relationship between HbA1c and stroke. The results showed that after adjusting age, gender, smoking, blood lipids and other variances, HbA1c and stroke risk was significantly associated. They emphasized that strict control of 
glycated hemoglobin (HbA1c) might be benefit for stroke prevention for the patients with diabetes. The present study showed that FPG, PPG values, NIHSS scores, on admission when compared among three groups of patients the difference was statistically significant $(\mathrm{P}<0.0001)$. Patients with high HbA1c, high FBG, high PPG had high NIHSS score at admission $(\mathrm{P}<0.001)$. That is to say a higher HbA1c level will have a more serious neurological impairment. So HbA1c levels at admission might be an important predictor to evaluate the neurological impairment aspects on admission, serious patients (> 15 NIHSS) of poor glycemic control accounted for $40 \%$ that is higher than good glycemic control (3.33\%) and non - diabetic (2.5\%).

The mechanism might be associated with long term high blood glucose and high blood HbA1c which lead to lesions of large blood vessels and which lead to shift oxygen dissociation curve to the left, resulting in oxygen dissociation barrier, nerve tissue ischemia and hypoxia, that Is not benefit for the recovery of neurological function, and the prognosis is worse, similar finding was noted by other studies $[15-18,19]$. In summary the current study suggests that blood HbA1c levels on admission may influence severity in patients with acute ischemic stroke. So HbA1c level may be is an important predictor to evaluate the neurological impairment in patients of acute ischemic stroke with diabetes.

Therefore, effectively lowering blood HbA1c levels may reduce the severity of neurological impairment in patients with acute ischemic stroke, and perhaps can improve the life quality of patient with ischemic stroke.

\section{Conclusion}

Among the diabetic stroke patient's severity of glycemic status found to have an influencing effect on the stroke severity. Estimation of $\mathrm{HbA1c}$ levels at the time of admission might be a predictor of the severity of neurological impairment in patients with acute ischemic stroke. With achievement of near normal glycemic status in diabetic population the stroke severity can be brought to the same extent of observed in non-diabetic population. We suggest the need for routine $\mathrm{HbA1c}$ testing in all patients with ischemic stroke for secondary prevention and routine screening in hospital registry for patients with high cardiovascular risk for primary prevention.

\section{References}

1. Wolf CD (2000) The impact of stroke. Br Med Bull 56(2): 275-286.

2. Adams HP, Davis PH, Leria EC, Chang KC, Bendixen BH, et al. (1999) Baseline NIH Stroke Scale score strongly predicts outcome after stroke: A report of the Trial of Org 10172 in Acute Stroke Treatment (TOAST). Neurology 53(1): 126-131.
3. Frankel MR, Morgenstern LB, Kwiatkowski T, Lu M, Tilley BC, et al. (2000) Predicting prognosis after stroke: a placebo group analysis from the national Institute of Neurological Disorders and Stroke rt_PA Stroke Trial. Neurology 55(7): 952-959.

4. Shindo A, Tomimoto H (2014) Diabetes and ischemic stroke. Brain Nerve 66: 107-119.

5. Cantu Brito C, Mimenza Alvarado A, Sanchez Hernandez JJ (2010) Diabetes mellitus and aging as a risk factor for cerebral vascular disease: epidemiology. Pathophysiology and prevention. Rev Invest Clin 62(4): 333-342.

6. Mankovsky BN, Ziegler D (2004) Stroke in patient with diabetes mellitus. Diabetes Metab Res Rev 20(4): 268-287.

7. Luitse MJ, Biessels GJ, Rutten GE, Kappelle LJ (2012) Diabetes, hyperglycaemia, and acute ischemic stroke. Lancet Neurol 11(3): 261271.

8. Kruyt ND, Biessels GJ, Devries JH, Roos YB (2010) Hyperglycemia in acute ischemic stroke: pathophysiology and clinical management. Nat Rev Neurol 6(3): 145-155.

9. Saudek CD, Derr RL, Kalyani RR (2006) Assessing glycemia in diabetes using self- monitoring blood glucose and hemoglobin A1c. JAMA 295(14): 1688-1697.

10. American Diabetes Association (2014) Standards of medical care in diabetes--2014. Diabetes Care 37(Suppl 1): S14-S80.

11. National Institute of Health, Institute of Neurological Disorder and Stroke. Stroke Scale.

12. Putaal J, Mesto AJ, Mesto TM, Konkola N, Kramemer Y, et al. (2009) Analysis of 1008 consecutive patients aged 15 to 49 with first - ever ischemic stroke, The Helsinki Young Stroke Registry. Stroke 40(4): 11951203.

13. Megherbi SE, Milan C, Minier D, Couvreur G, Osseby GV, et al. (2003) Association between diabetes and stroke subtype on survival and functional outcome 3 months after stroke: Data from the European Biomed Stroke Project. Stroke 34(3): 688-694.

14. Kizer JR, Wibers DO, Whisnant JP, Galloway JM, Welty TK, et al. (2006) Glycerinic level and future stroke in type 2 diabetes mellitus: The strong heart study. Circulation 114: 444-446.

15. Kamouchi M, Matsuki T, Hata J, Kuwashiro T, Ago T, et al. (2011) Pre stroke glycemic control is associated with the functional out come in acute ischemic stroke: the Fukuoka Stroke Registry. Stroke 42(10): 2788-2794.

16. Hjalmarsson C, Manhem K, Bokemark L, Andersson B (2014) The role of pre-stroke control on severity and outcome of acute ischemic stroke. Stroke Res Treat 2014: 694569.

17. Wu S, Wang C, Jia Q Liu G, Hoff $\mathrm{K}$, et al. (2014) HbA1c is as associated with increased all - cause mortality in the first year after acute ischemic stroke. Neurol Res 36(5): 444-452.

18. Lei C, Wu B, Liu M, Chen Y (2015) Association between hemoglobin A1c level and clinical outcome in ischemic stroke patients with or without diabetes. J Clin Neurosci 22(3): 498-503. 